\title{
Erbium-Doped Laser with Multi-segmented Silicon Nitride Structure
}

\author{
Purnawirman, ${ }^{1, *}$ E. Shah Hosseini, ${ }^{1}$ A. Baldycheva, ${ }^{1}$ J. Sun, ${ }^{1}$ J. D. B. Bradley, ${ }^{1}$ T. N. Adam, ${ }^{2}$ G. Leake, ${ }^{2}$ \\ D. Coolbaugh, ${ }^{2}$ and M. R. Watts ${ }^{1}$ \\ ${ }^{1}$ Photonic Microsystems Group, Research Laboratory of Electronics, Massachusetts Institute of Technology, \\ 77 Massachusetts Avenue, Cambridge, Massachusetts 02139, USA \\ ${ }^{2}$ College of Nanoscale Science and Engineering, University at Albany, State University of New York, \\ 257 Fuller Road, Albany, New York 12203, USA \\ *Corresponding author: purna@mit.edu
}

\begin{abstract}
We report on DFB and DBR lasers formed from a wavelength insensitive multisegmented silicon nitride waveguide. Using a five-segment waveguide, we obtain lasing in erbium-doped DBR (-3.6 dBm) and DFB (-7.3 dBm) cavities.

OCIS codes: (130.0130) Integrated optics; (130.2790) Guided waves; (130.3120) Integrated optics devices; (140.3460)

Lasers.
\end{abstract}

\section{Introduction}

Lasers on a silicon platform have generated substantial interest due to the numerous important applications ranging from integrated communications to optical sensors. Several methods have been developed to realize on-chip lasers, including germanium-on-silicon lasers [1], III-V hybrid lasers [2, 3], and erbium-doped glass lasers [4, 5]. Of these approaches, only erbium-doped glass lasers have been shown to achieve both ultra-narrow linewidth [4] and CMOScompatibility [3]. Thus, the next step is to demonstrate integration with other photonics devices. In particular, silicon nitride based waveguides provide robust, low-loss and compact devices for a wide range of optical wavelengths [6, 7]. Erbium laser resonators have been demonstrated by patterning silicon nitride structures beneath the erbium host material $\left(\mathrm{Al}_{2} \mathrm{O}_{3}\right)$ to form an inverted ridge waveguide [4, 5]. In a traditional waveguide design, the relatively high index contrast of these materials $\left(n_{\mathrm{SiN}_{x}}=1.99\right.$ and $\left.n_{\mathrm{Al}_{2} \mathrm{O}_{3}}=1.65\right)$ requires that the silicon nitride layer be very thin so that the mode overlaps strongly with the gain medium. However, a thin silicon nitride layer prevents the layer from being effectively reused to form other important integrated devices such as microring-resonators.

Here, we solve this problem by implementing a multi-segmented silicon nitride waveguide [8] to achieve strong overlap with the active region while maintaining a thick silicon nitride layer for passive component integration. Additionally, the resulting design facilitates broadband, high confinement and overlap factors across the entire nearinfrared wavelengths $(0.9 \mu \mathrm{m}-2.0 \mu \mathrm{m})$ offering potential for amplifier and laser operation across an extremely broad bandwidth, a result that will become of increasing importance as the $\mathrm{S}, \mathrm{C}$, and $\mathrm{L}$ bands become saturated. We demonstrate multi-segmented waveguide $\mathrm{Er}_{3+}: \mathrm{Al}_{2} \mathrm{O}_{3}$ lasers in distributed Bragg reflector (DBR) cavities with maximum output powers of $-3.6 \mathrm{dBm}$ and distributed feedback (DFB) cavities with maximum output powers of -7.3 $\mathrm{dBm}$ at a wavelength of $\lambda \sim 1565 \mathrm{~nm}$.

\section{Design and Simulation}

Figure 1 a) shows the schematic of the multi-segmented waveguide designs for $\mathrm{Er}_{3+}: \mathrm{Al}_{2} \mathrm{O}_{3}$ lasers. The waveguide consists of a silicon $(\mathrm{Si})$ substrate, a five segment $\mathrm{SiN}_{\mathrm{x}}$ waveguide buried in $\mathrm{SiO}_{2}\left(n_{\mathrm{SiO}_{2}}=1.445\right)$, and a $\mathrm{SiO}_{2}$ gap between the top surface of the $\mathrm{SiN}_{\mathrm{x}}$ and the $\mathrm{Er}_{3}: \mathrm{Al}_{2} \mathrm{O}_{3}$. The distance between the $\mathrm{Si}$ to the bottom layer of $\mathrm{SiN}_{\mathrm{x}}$ $(>2.5 \mu \mathrm{m})$ was chosen so that the fundamental TE mode is not affected by the substrate. The parameters of the $\mathrm{SiN}_{\mathrm{x}}$ waveguide consist of a waveguide thickness $t=200 \mathrm{~nm}$, width $w=600 \mathrm{~nm}$, and gap $g=400 \mathrm{~nm}$. A layer of oxide $\left(\mathrm{SiO}_{2}\right)$ filling the gap with thickness of $200 \mathrm{~nm}$ was deposited in between the erbium glass and $\mathrm{SiN}_{\mathrm{x}}$ to help reduce the guiding effect of high index $\mathrm{SiN}_{\mathrm{x}}$ [5]. Lastly, an $\mathrm{Er}_{3+}: \mathrm{Al}_{2} \mathrm{O}_{3}$ of thickness of $1500 \mathrm{~nm}$ was put on top of the structure.

The intensity distributions of the fundamental TE mode at different wavelengths were calculated by a finite difference mode solver. Figure $1 \mathrm{~b}$ ) and c) show the intensity distribution for pump wavelength at $980 \mathrm{~nm}$ and signal wavelength at $1550 \mathrm{~nm}$. If we define the confinement factor $(\gamma)$ and overlap factor $(\Gamma)$ in the active medium by the following, 


$$
\begin{gathered}
I_{s / p}=\frac{A}{I_{s / p} d A}=\frac{I_{i c t i v e(i j)} I_{i j}^{(s / p)}}{I_{i j}^{(s / p)}} \\
\Gamma_{s p}=\frac{\int_{A} I_{p} I_{s} d A}{\sqrt{\int_{A} I_{p}^{2} d A \int_{A} I_{s}^{2} d A}}=\frac{\sum_{\text {active }(i j)} I_{i j}^{(p)} I_{i j}^{(s)}}{\sqrt{\sum_{\text {active }(i j)} I_{i j}^{2(p)}} \sqrt{\sum_{\text {active }(i j)} I_{i j}^{2(s)}}}
\end{gathered}
$$

then we obtain $\gamma_{1550 \mathrm{~nm}}=90 \%, \gamma_{980 \mathrm{~nm}}=89 \%$, and $\Gamma_{980-1550}=99 \%$.

a) Air

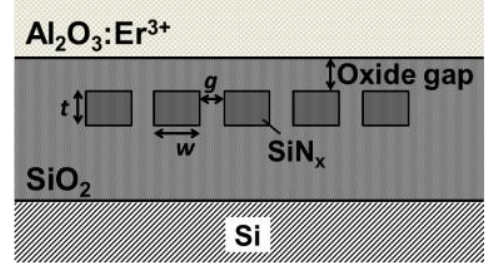

b)
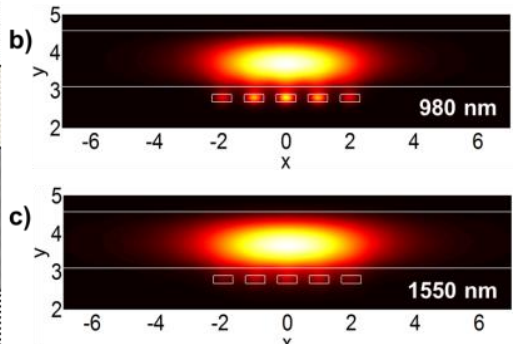

Figure 1 a) Schematic of the vertical five-segment waveguide design. The calculated intensity of fundamental TE mode at b) $980 \mathrm{~nm}$ (pump) and c) $1550 \mathrm{~nm}$ (signal).

We extended our analysis of the multi-segmented structure to broader wavelength selections; $950 \mathrm{~nm}$ and 980 $\mathrm{nm}$ (InGaAs pump diode), $1050 \mathrm{~nm}$ (Ytterbium/Yb and Neodymium/Nd doped laser), $1300 \mathrm{~nm}$ (Nd doped laser), $1550 \mathrm{~nm}$ (Er doped laser), and $2000 \mathrm{~nm}$ (Thulium/Tm and Holmium/Ho doped laser). The confinement and overlap factors (with $980 \mathrm{~nm}$ as the reference pump) of these wavelengths are shown in Figure 2. We obtained $>85 \%$ confinement factor for all wavelengths longer than $950 \mathrm{~nm}$ and $>98 \%$ intensity overlap factor with $980 \mathrm{~nm}$ pump over the entire near-infrared wavelength light sources, demonstrating insensitivity of mode profiles at various wavelengths. We note that the calculation has ignored the variation of the refractive indices at various wavelengths.
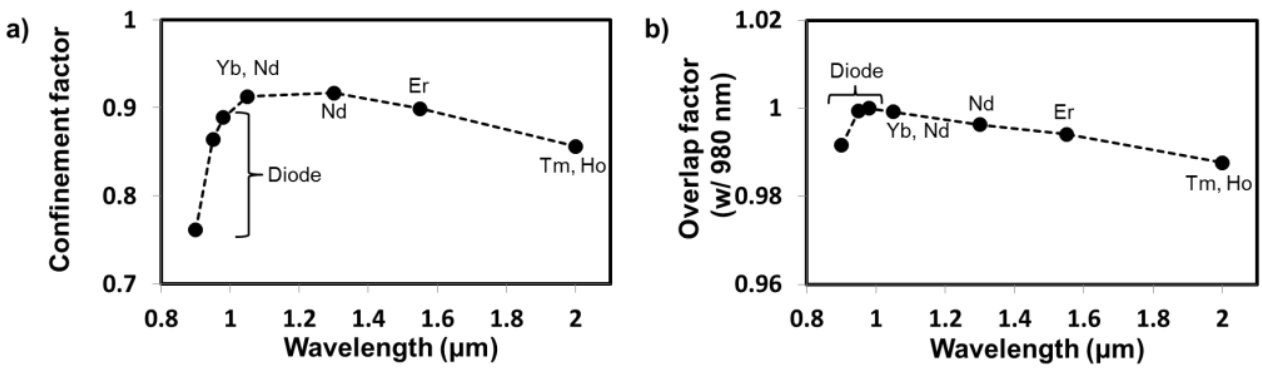

Figure 2 a) Confinement factor of different light sources. b) Intensity overlap factor of different wavelength with $980 \mathrm{~nm}$.

\section{3. $\mathrm{Er}_{3+}: \mathrm{Al}_{2} \mathrm{O}_{3}$ Laser based on $\mathrm{VMS}$ waveguide}

We designed DBR and DFB resonators for $\mathrm{Er}_{3+}: \mathrm{Al}_{2} \mathrm{O}_{3}$ lasers. Periods of both DBR and DFB are the same $\Lambda=504$ $\mathrm{nm}$. The DBR cavity consists of a straight multi-segmented waveguide of length $L_{\text {gain }}=13.8 \mathrm{~mm}$ confined by two similar reflection gratings. Each grating is formed by periodic additional side pieces on both sides of the multisegmented waveguide (adding to total of $7 \mathrm{SiN}_{\mathrm{x}}$ segment waveguides), with each additional pieces having widths of $w_{D B R}=600 \mathrm{~nm}$ and separated from the multi-segmented waveguide by a gap of $g_{D B R}=400 \mathrm{~nm}$. The total length of each of the gratings is given by $L_{D B R}=5 \mathrm{~mm}$. The DFB cavity consists of grating across the entire gain region, with the grating also formed by periodic additional side pieces with $w_{D F B}=300 \mathrm{~nm}$ and $g_{D F B}=550 \mathrm{~nm}$. The total length of the DFB is $L_{D F B}=20 \mathrm{~mm}$. The quarter-wave phase shift of the DFB was designed to be slightly off from the center of the cavity $\left(0.6 L_{D F B}\right)$ so as to ensure lasing out of a single facet.

The laser cavities were fabricated within a 300mm CMOS foundry. A $6 \mu \mathrm{m}$-thick plasma-enhanced chemical vapor deposition (PECVD) $\mathrm{SiO}_{2}$ layer with $0.2 \mu \mathrm{m}$-thick $\mathrm{LPCVD} \mathrm{SiN}_{\mathrm{x}}$ layer was first purchased from vendor. The nitride layer was then patterned using $193 \mathrm{~nm}$ immersion lithography and reactive ion etching. After patterning, a PECVD $\mathrm{SiO}_{2}$ layer was deposited and chemically mechanically polished (CMP)-ed to a height of $0.1 \mu \mathrm{m}$ above the silicon nitride layer. A second nitride layer was then deposited and patterned with a thickness of $0.2 \mu \mathrm{m}$ and buried 
under additional $4 \mu \mathrm{m} \mathrm{SiO}_{2}$ layer. The second nitride layer is a part of process for other devices, however in the case of laser structures, we utilized this layer as an etch stop for erbium trench. After the nitride was etched away, we deposited another $0.1 \mu \mathrm{m} \mathrm{SiO}$ to make the total oxide gap to become $0.2 \mu \mathrm{m}$. Trenches for dicing and fiber end coupling were then etched into the edges of the dies by deep oxide and silicon etching. Finally, the wafers were transferred from the CMOS foundry, diced into individual dies, and an $\mathrm{Er}_{3+}: \mathrm{Al} 2 \mathrm{O} 3$ layer was deposited by reactive co-sputtering using a process similar to that reported in [9]. Using the prism coupling method to measure the planar losses around $1550 \mathrm{~nm}$, we obtained background loss, dopant concentration, and thickness of the film to be $<0.1$ $\mathrm{dB} / \mathrm{cm}, 1.0 \times 10^{20} \mathrm{~cm}-3$, and $1 \mu \mathrm{m}$ respectively. The confinement and overlap factors for $1 \mu \mathrm{m}$-thick $\mathrm{Er}_{3} \div \mathrm{Al}_{2} \mathrm{O}_{3}$ are given by $\gamma_{1550 \mathrm{~nm}}=75 \%, \gamma_{1550 \mathrm{~nm}}=66 \%$, and $\Gamma_{980-1550}=99 \%$ respectively.
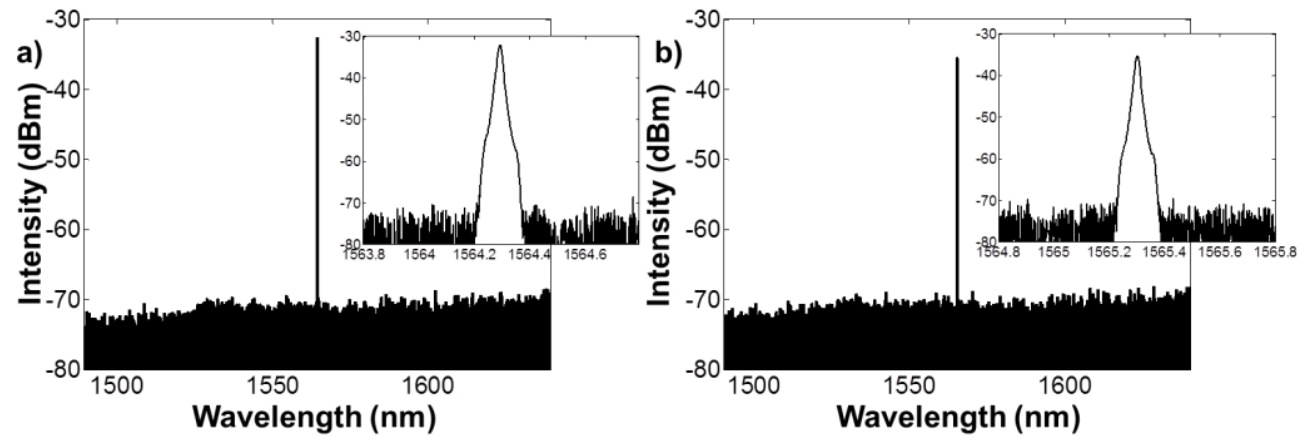

Figure 3 Spectrum of a) DBR and b) DFB lasers.

The DBR and DFB lasers were pumped by using two $978 \mathrm{~nm}$ diode lasers. By transmission measurement, we measured the coupling losses. For the DBR laser, we estimated maximum on-chip power of $P_{\max }=0.44 \mathrm{~mW}(-3.6$ $\mathrm{dBm}$ ) and slope efficiency of $\eta=1.4 \%$. The threshold pump power is $P_{t h}=64 \mathrm{~mW}$. For DFB laser, we estimated maximum on-chip power of $P_{\max }=0.18 \mathrm{~mW}(-7.3 \mathrm{dBm})$ and slope efficiency of $\eta=2.7 \%$. The threshold pump power is $P_{t h}=14 \mathrm{~mW}$. Figure 3 shows the spectrum of the DBR and DFB lasers at $\sim 1565 \mathrm{~nm}$.

\section{Conclusion}

We report on a wavelength insensitive design for the near-infrared range by using a multi-segmented waveguide design. The design allows for high confinement and intensity overlap between the pump and signal wavelengths. We use a five-segmentwaveguide design to demonstrate $\mathrm{Er}_{3}: \mathrm{Al}_{2} \mathrm{O}_{3}$ in DBR and DFB cavities. We obtained maximum power of $-3.6 \mathrm{dBm}$ for DBR laser and $-7.3 \mathrm{dBm}$ for DFB laser. The waveguide design allows operation of lasers at different wavelengths and due to the increased layer thickness, a further integration of compact silicon nitride devices.

This work was funded by the Defense Advanced Research Projects Agency Electronic Photonics Integration (DARPA-EPHI) program, grant no. HR0011-12-2-0007 and the Samsung Global Research Outreach (GRO) program.

\section{References}

1. Camacho-Aguilera, R.E., et al., An electrically pumped germanium laser. Optics Express, 2012. 20(10): p. 11316-11320.

2. Srinivasan, S., et al., Design of phase-shifted hybrid silicon distributed feedback lasers. Optics Express, 2011. 19(10): p. 9255-9261.

3. Sun, X.K., et al., Electrically pumped hybrid evanescent Si/InGaAsP lasers. Optics Letters, 2009. 34(9): p. 1345-1347.

4. Bernhardi, E.H., et al., Ultra-narrow-linewidth, single-frequency distributed feedback waveguide laser in Al2O3:Er3+ on silicon. Optics Letters, 2010. 35(14): p. 2394-2396.

5. $\quad$ Purnawirman, et al., C- and L-band erbium-doped waveguide lasers with wafer-scale silicon nitride cavities. Optics Letters, 2013. 38(11): p. 1760-1762.

6. Gondarenko, A., J.S. Levy, and M. Lipson, High confinement micron-scale silicon nitride high Q ring resonator. Optics Express, 2009. 17(14): p. 11366-11370.

7. Hosseini, E.S., et al., High Quality Planar Silicon Nitride Microdisk Resonators for Integrated Photonics in the Visible Wavelength Range. Optics Express, 2009. 17(17): p. 14543-14551.

8. Vivien, L., et al., Vertical multiple-slot waveguide ring resonators in silicon nitride. Optics Express, 2008. 16(22): p. $17237-17242$.

9. Worhoff, K., et al., Reliable Low-Cost Fabrication of Low-Loss A12O3:Er3+ Waveguides With 5.4-dB Optical Gain. Ieee Journal of Quantum Electronics, 2009. 45(5-6): p. 454-461. 